\title{
Pacific
}

Journal of

Mathematics

\section{REPRESENTATIONS OF LIE SUPERALGEBRAS IN PRIME CHARACTERISTIC, III}

\section{LEI ZHAO}




\title{
REPRESENTATIONS OF LIE SUPERALGEBRAS IN PRIME CHARACTERISTIC, III
}

\author{
LEI ZHAO
}

\begin{abstract}
For a restricted Lie superalgebra $\mathfrak{g}$ over an algebraically closed field of characteristic $p>2$, we generalize the deformation method of Premet and Skryabin to obtain results on the $p$-power and 2-power divisibility of dimensions of $\mathfrak{g}$-modules. In particular, we give a new proof of the super Kac-Weisfeiler conjecture for basic classical Lie superalgebras. The new proof allows us to improve optimally the assumption on $p$. We also establish a semisimplicity criterion for the reduced enveloping superalgebras associated with semisimple $p$-characters for all basic classical Lie superalgebras using the technique of odd reflections.
\end{abstract}

\section{Introduction}

In [Wang and Zhao 2009a], Wang and the author initiated the study of modular representation theory of Lie superalgebras over an algebraically closed field $K$ of characteristic $p>2$. Among other things, we formulated a superalgebra generalization (called the super KW conjecture) of the celebrated Kac-Weisfeiler conjecture (Premet's theorem), and we established it for the most important class of Lie superalgebras - the basic classical Lie superalgebras, which were first classified over the complex numbers by Kac [1977] and Scheunert, Nahm, and Rittenberg [1976]. Our work generalized the earlier work on Lie algebras of reductive algebraic groups by Veŭsfeřler and Kac [1971], Friedlander and Parshall [1988], Premet [1995; 2002] Skryabin [2003], and others. See [Jantzen 1998] for an excellent review and extensive references on modular representations of Lie algebras.

In our proof of the super KW conjecture, a $\mathbb{Z}$-grading of the basic classical Lie superalgebras plays an important role. To obtain the grading, we imposed somewhat restrictive conditions on $p$ [Wang and Zhao 2009a, Section 2.2].

To derive results on dimensions of simple $\mathscr{L}$-modules, Premet and Skryabin [1999] developed deformation techniques by considering a family of $\mathscr{L}$-associative algebras for a restricted Lie algebra $\mathscr{L}$. In particular, their method gives a new proof

MSC2000: primary 17B10, 17B50; secondary 17B20.

Keywords: Lie superalgebras, modular representations. 
of the Kac-Weisfeiler conjecture that differs completely from Premet's original approach [1995].

Our first main goal here is to generalize some of the ideas in [Premet and Skryabin 1999] to the superalgebra setting. In particular, we provide a new proof of the super KW conjecture for basic classical Lie superalgebras, so that the overly restrictive assumption on $p$ in [Wang and Zhao 2009a, Section 2.2] is relaxed optimally.

Our second goal is to give a simplicity criterion for baby Verma modules as well as a semisimplicity criterion for reduced enveloping superalgebras of basic classical Lie superalgebras with semisimple $p$-characters.

Let $\mathfrak{g}=\mathfrak{g}_{0}+\mathfrak{g}_{\overline{1}}$ be an $\left(n_{0} \mid n_{1}\right)$-dimensional restricted Lie superalgebra over $K$ and let $\xi \in \mathfrak{g}_{\overline{0}}^{*}$. Let $S(\mathfrak{g})$ be the symmetric superalgebra on $\mathfrak{g}$. The reduced symmetric superalgebra $S_{\xi}(\mathfrak{g})$ associated with $\xi$ is defined to be the quotient of $S(\mathfrak{g})$ by the ideal generated by elements of the form $(x-\xi(x))^{p}$ with $x \in \mathfrak{g}_{\overline{0}}$. It is a local (super)commutative superalgebra of dimension $p^{n_{0}} 2^{n_{1}}$. Let $U_{\xi}(\mathfrak{g})$ be the reduced enveloping superalgebra as usual.

Following Premet and Skryabin [1999], we introduce a family $U_{\xi, \lambda}(\mathfrak{g})$ of associative superalgebras, where $\xi \in \mathfrak{g}_{0}^{*}$ and $\lambda \in K$, parametrized by the points of the projective space $\mathbb{P}\left(\mathfrak{g}_{0}^{*} \oplus K\right)$, the superalgebras $U_{t \xi, t \lambda}(\mathfrak{g})$ with $t \in K^{\times}$being isomorphic. The Lie superalgebra $\mathfrak{g}$ acts on each $U_{\xi, \lambda}(\mathfrak{g})$ as derivations. The family relates the reduced enveloping superalgebra $U_{\xi}(\mathfrak{g})\left(=U_{\xi, 1}(\mathfrak{g})\right)$ to the reduced symmetric superalgebra $S_{\xi}(\mathfrak{g})\left(=U_{\xi, 0}(\mathfrak{g})\right)$. As in their Lie algebra case, $S_{\xi}(\mathfrak{g})$ has favorable structures of $\mathfrak{g}$-invariant ideals (see Proposition 2.6).

Again following Premet and Skryabin [1999] but with slight modification, we use the method of associated cones in invariant theory to obtain some results on the $(p, 2)$-divisibility of dimensions of $\mathfrak{g}$-modules. In particular, we show in Theorem 3.2(ii) that for an arbitrary restricted Lie superalgebra $\mathfrak{g}$ and $\chi \in \mathfrak{g}_{0}^{*}$, if

( $\star$ all nonzero scalar multiples of $\chi$ are conjugate under the group $G\left(\mathfrak{g}_{0}\right)$ of automorphisms of $\mathfrak{g}_{0}$ that preserve the restricted structure,

then the super KW conjecture holds for $U_{\chi}(\mathfrak{g})$. Note that $(\star)$ is a nonsuper condition. If $\mathfrak{g}$ is one of the basic classical Lie superalgebras of Section $2 \mathrm{~d}$ with the optimal assumption on $p$ or the queer Lie superalgebra of [Wang and Zhao 2009b] and if $\chi \in \mathfrak{g}_{0}^{*}$ is nilpotent, then ( $\star$ ) is satisfied [Jantzen 2004, Sections 2.8, 2.10]. Thus the super KW conjecture for basic classical Lie superalgebras and the queer Lie superalgebra with nilpotent $p$-characters holds. Together with the Morita equivalence theorem [Wang and Zhao 2009a, Theorem 5.2], this gives a new proof of the super KW conjecture for basic classical Lie superalgebras in full generality with the optimal assumption on $p$. 
For the reduced enveloping superalgebras of basic classical Lie superalgebras with semisimple $p$-characters, we give a simplicity criterion for baby Verma modules, and hence obtain a semisimplicity criterion for the reduced enveloping superalgebras. These results, first announced in [Zhao 2009, Remark 4.5], generalize results of Rudakov [1970] and Friedlander and Parshall [1988] for Lie algebras.

A major complication in the super case is due to the existence of nonconjugate sets of simple roots. We settle the problem by using the technique of odd reflections (see [Serganova 2008] for example). This approach is quite different from the proof of the corresponding results for type I basic classical Lie superalgebras in [Zhao 2009].

C. Zhang [2009] independently stated the simplicity criterion for baby Verma modules with semisimple $p$-characters for basic classical Lie superalgebras (the statement of Theorem 4.6). However, his proof, which relied essentially on an erroneous lemma [Zhang 2009, Lemma 3.6], is incorrect.

The paper is laid out as follows. In Section 2, after reviewing some basic facts about modular representations of Lie superalgebras and basic classical Lie superalgebras, we introduce the super generalization of families of associative algebras following [Premet and Skryabin 1999]. Then we study the properties of invariant ideals of the reduced symmetric superalgebras. The new proof of super KW conjecture for basic classical Lie superalgebras is given in Section 3. Section 4 is devoted to the study of basic classical Lie superalgebras with semisimple $p$-characters.

\section{Restricted Lie superalgebras and families of $\mathfrak{g}$-superalgebras}

2a. Throughout we work with an algebraically closed field $K$ with characteristic $p>2$ as the ground field. We exclude $p=2$ since in that case Lie superalgebras coincide with Lie algebras.

A superspace is a $\mathbb{Z}_{2}$-graded vector space $V=V_{\overline{0}} \oplus V_{\overline{1}}$, in which we call elements in $V_{\overline{0}}$ even and those in $V_{\overline{1}}$ odd. Write $|v| \in \mathbb{Z}_{2}$ for the parity (or degree) of $v \in V$, which is implicitly assumed to be $\left(\mathbb{Z}_{2}\right.$-)homogeneous. A bilinear form $f$ on $V$ is supersymmetric if $f(u, v)=(-1)^{|u||v|} f(v, u)$ for all homogeneous $u, v \in V$. We will use the notation

$$
\underline{\operatorname{dim}} V=\operatorname{dim} V_{\overline{0}} \mid \operatorname{dim} V_{\overline{1}} \quad \text { and } \quad \operatorname{dim} V=\operatorname{dim} V_{\overline{0}}+\operatorname{dim} V_{\overline{1}} .
$$

If $W$ is a subsuperspace of $V$, write

$$
\underline{\operatorname{codim}}_{V} W=\underline{\operatorname{dim}} V-\underline{\operatorname{dim}} W \quad \text { and } \quad \operatorname{codim}_{V} W=\operatorname{dim} V-\operatorname{dim} W .
$$

Sometimes we simply write $\operatorname{codim} W$ and $\operatorname{codim} W$ for short when the total space $V$ is clear from the context. 
All Lie superalgebras $\mathfrak{g}$ will be assumed to be finite-dimensional. We will use $U(\mathfrak{g})$ to denote its universal enveloping superalgebra.

According to Walls [1964], the finite-dimensional simple associative superalgebras over $K$ are classified into two types: besides the usual matrix superalgebra (called type $M$ ) there are also simple superalgebras of type $Q$.

We speak of vector spaces, derivations, subalgebras, ideals, modules, submodules, and commutativity, and so on in the super sense unless otherwise specified.

For a real number $a$, we use $\lfloor a\rfloor$ to denote its least integer upper bound, and use $\lceil a\rceil$ to denote its greatest integer lower bound.

2b. A restricted Lie superalgebra $\mathfrak{g}=\mathfrak{g}_{\overline{0}} \oplus \mathfrak{g}_{\overline{1}}$ is a Lie superalgebra whose even subalgebra $\mathfrak{g}_{0}$ is a restricted Lie algebra that admits a $[p]$-th power map ${ }^{[p]}: \mathfrak{g}_{\overline{0}} \rightarrow \mathfrak{g}_{0}$ satisfying certain conditions [Jacobson 1962, Chapter V], and whose odd part $\mathfrak{g}_{\overline{1}}$ is a restricted module by the adjoint action of the even subalgebra $\mathfrak{g}_{0}$.

All the Lie (super)algebras in this paper will be assumed to be restricted.

Let $\mathfrak{g}$ be a restricted Lie superalgebra. For each $\chi \in \mathfrak{g}_{0}^{*}$, the reduced enveloping superalgebra of $\mathfrak{g}$ with the $p$-character $\chi$ is by definition the quotient of $U(\mathfrak{g})$ by the ideal $I_{\chi}$ generated by all $x^{p}-x^{[p]}-\chi(x)^{p}$ with $x \in \mathfrak{g}_{\overline{0}}$.

We further recall the definition of (super)derivations. Let $A=A_{\overline{0}} \oplus A_{\overline{1}}$ be an associative superalgebra. Then its endomorphism algebra $\operatorname{End}_{K}(A)$ is naturally $\mathbb{Z}_{2}$-graded with

$$
\operatorname{End}_{K}(A)_{i}=\left\{f \in \operatorname{End}_{K}(A) \mid f\left(A_{j}\right) \subseteq A_{j+i} \text { for } j \in \mathbb{Z}_{2}\right\} \quad \text { for } i \in \mathbb{Z}_{2} .
$$

For $i \in \mathbb{Z}_{2}$, let $\operatorname{Der}_{i}(A)$ be the subspace of all $\delta \in \operatorname{End}_{K}(A)_{i}$ such that

$$
\delta(x y)=(\delta x) y+(-1)^{i|x|} x(\delta y) \text { for all homogeneous } x, y \in A .
$$

The Lie superalgebra of derivations of $A$, that is, $\operatorname{Der}(A)=\operatorname{Der}_{\overline{0}}(A) \oplus \operatorname{Der}_{\overline{1}}(A)$, is a restricted Lie subalgebra of $\operatorname{End}_{K}(A)$.

2c. Let $\mathfrak{g}$ be a restricted Lie superalgebra. For $\chi \in \mathfrak{g}_{0}^{*}$, we always regard $\chi$ as being in $\mathfrak{g}^{*}$ by setting $\chi\left(\mathfrak{g}_{\overline{1}}\right)=0$. Denote the centralizer of $\chi$ in $\mathfrak{g}$ by $\mathfrak{g}_{\chi}=\mathfrak{g}_{\chi, \overline{0}}+\mathfrak{g}_{\chi, \overline{1}}$, where $\mathfrak{g}_{\chi, i}=\left\{y \in \mathfrak{g}_{i} \mid \chi([y, \mathfrak{g}])=0\right\}$ for $i \in \mathbb{Z}_{2}$. Set $d_{0} \mid d_{1}=\underline{\text { codim }} \mathfrak{g}_{\chi}$. It is well known that $d_{0}$ is even whereas $d_{1}$ could be odd.

We recall here the superalgebra generalization of the Kac-Weisfeiler conjecture.

Super KW conjecture [Wang and Zhao 2009a]. The dimension of every $U_{\chi}(\mathfrak{g})$ module is divisible by $p^{d_{0} / 2} 2^{\left\lfloor d_{1} / 2\right\rfloor}$.

2d. The basic classical Lie superalgebras over the complex field $\mathbb{C}$ were classified independently by Kac [1977] and Scheunert, Nahm and Rittenberg [1976]. Those Lie superalgebras by definition admit an even nondegenerate supersymmetric bilinear form, and the even subalgebras are reductive. 


\begin{tabular}{cc}
\hline Lie superalgebra & characteristic of $K$ \\
\hline $\mathfrak{g l}(m \mid n)$ & $p>2$ \\
$\mathfrak{s l}(m \mid n)$ & $p>2, p \nmid(m-n)$ \\
$B(m, n), C(n), D(m, n)$ & $p>2$ \\
$D(2,1 ; \alpha)$ & $p>3$ \\
$F(4)$ & $p>2$ \\
$G(3)$ & $p>3$ \\
\hline
\end{tabular}

Table 1. Basic classical Lie $K$-superalgebras.

The basic classical Lie superalgebras are also defined over fields of positive characteristics under mild assumptions on $p$; see [Wang and Zhao 2009a, Section 2]. The restriction on the characteristic of fields of definition is listed in Table 1. The general linear Lie superalgebra, though not simple, is also included.

For each basic classical Lie superalgebra $\mathfrak{g}$, the restriction on the prime $p$ in the table makes $p$ automatically good for the even subalgebra $\mathfrak{g}_{0}^{-}$; see [Jantzen 2004, Section 2.6].

2e. In the next two subsections, we follow Premet and Skryabin [1999] by introducing a family of associative superalgebras deformed from the reduced enveloping superalgebras. This part can be viewed as a super counterpart of their Section 2; since the proofs of the statements are essentially the same as the corresponding ones in their paper, we will omit them.

Let $\mathfrak{g}$ be a $\left(n_{0} \mid n_{1}\right)$-dimensional restricted Lie superalgebra. A $\mathfrak{g}$-superalgebra is a pair consisting of a $K$-superalgebra $A$ and a homomorphism $\mathfrak{g} \rightarrow \operatorname{Der} A$ of restricted Lie superalgebras.

Given a linear form $\xi \in \mathfrak{g}_{0}^{*}$ and a scalar $\lambda \in K$, denote by $U_{\xi, \lambda}(\mathfrak{g})$ the quotient superalgebra of the tensor superalgebra $T(\mathfrak{g})$ on the superspace $\mathfrak{g}$ by its ideal $I_{\xi, \lambda}$ generated by all elements $x \otimes y-(-1)^{|x||y|} y \otimes x-\lambda[x, y]$ for all homogeneous $x, y \in \mathfrak{g}$ and elements $x^{\otimes p}-\lambda^{p-1} x^{[p]}-\xi(x)^{p} \cdot 1$ for all $x \in \mathfrak{g}_{0}$. Each $U_{\xi, \lambda}(\mathfrak{g})$ is a $\mathfrak{g}$-superalgebra.

If $\lambda=1$, the superalgebra $U_{\xi, \lambda}(\mathfrak{g})$ is the reduced enveloping superalgebra $U_{\xi}(\mathfrak{g})$, while if $\lambda=0$, the superalgebra is called the reduced symmetric superalgebra, denoted by $S_{\xi}(\mathfrak{g})$. Since $x^{p}-\xi(x)^{p}=(x-\xi(x))^{p}$ for $x \in \mathfrak{g}_{0}$, by change of variables we see that $S_{\xi}(\mathfrak{g})$ is isomorphic to the truncated polynomial superalgebra

$$
K\left[x_{1}, \ldots, x_{n_{0}} ; y_{1}, \ldots, y_{n_{1}}\right] /\left(x_{1}^{p}, \ldots, x_{n_{0}}^{p} ; y_{1}^{2}, \ldots, y_{n_{1}}^{2}\right),
$$

where $K\left[x_{1}, \ldots, x_{n_{0}} ; y_{1}, \ldots, y_{n_{1}}\right]$ is the (free) commutative superalgebra on even generators $\left\{x_{1}, \ldots, x_{n_{0}}\right\}$ and odd generators $\left\{y_{1}, \ldots, y_{n_{1}}\right\}$. The unique maximal ideal of $S_{\xi}(\mathfrak{g})$ is generated by all $x-\xi(x) \cdot 1$ for $x \in \mathfrak{g}_{\overline{0}}$ and all $y \in \mathfrak{g}_{\overline{1}}$. 
If $t \in K^{\times}=K \backslash\{0\}$, the map $x \mapsto t^{-1} x$, where $x \in \mathfrak{g}$, extends uniquely to the superalgebra isomorphism $\theta_{t}: U_{\xi, \lambda}(\mathfrak{g}) \rightarrow U_{t \xi, t \lambda}(\mathfrak{g})$. In particular, if $\lambda \neq 0$, then $U_{\xi, \lambda}(\mathfrak{g}) \cong U_{\lambda^{-1} \xi}(\mathfrak{g})$ as superalgebras. All superalgebra isomorphisms $\theta_{t}$ are $\mathfrak{g}$-equivariant.

2f. A vector bundle $A \rightarrow Z$ over an algebraic variety $Z$ together with a pair of morphism $\mu: A \times_{Z} A \rightarrow A$ and $\rho: \mathfrak{g} \times A \rightarrow A$ of algebraic varieties over $Z$ is called a continuous family of (finite-dimensional) $\mathfrak{g}$-superalgebras parametrized by $Z$ if, for the fiber $A_{\zeta}$ over any point $\zeta \in Z$,

(1) the restriction of $\mu$ to $A_{\zeta} \times A_{\zeta}$ gives $A_{\zeta}$ a structure of a finite-dimensional associative superalgebra, and

(2) the restriction of $\rho$ to $\mathfrak{g} \times A_{\zeta}$ induces a homomorphism of restricted Lie superalgebras $\mathfrak{g} \rightarrow \operatorname{Der} A_{\zeta}$.

The algebraic variety $Z$ is called the parameter space of the family. By definition, all $\mathfrak{g}$-superalgebras in a family have the same finite dimension.

The isomorphisms $\theta_{t}$ allow us pass to a continuous family of superalgebras parametrized by the projective space $\mathbb{P}\left(\mathfrak{g}_{0}^{*} \oplus K\right)$ corresponding to the linear space $\mathfrak{g}_{0}^{*} \oplus K$. Write $(\xi: \lambda)$ for the point of $\mathbb{P}\left(\mathfrak{g}_{0}^{*} \oplus K\right)$ that is represented by the pair $(\xi, \lambda) \neq(0,0)$, where $\xi \in \mathfrak{g}_{0}^{*}, \lambda \in K$. Identify $\mathbb{P}\left(\mathfrak{g}_{0}^{*}\right)$ with the Zariski closed subset of $\mathbb{P}\left(\mathfrak{g}_{0}^{*} \oplus K\right)$ consisting of all points $(\xi: \lambda)$ with $\lambda=0$. Identify each $\xi \in \mathfrak{g}_{0}^{*}$ with the point $(\xi: 1) \in \mathbb{P}\left(\mathfrak{g}_{0}^{*} \oplus K\right)$.

Proposition 2.1. The set of superalgebras $U_{\xi, \lambda}(\mathfrak{g})$ with $(\xi: \lambda) \in \mathbb{P}\left(\mathfrak{g}_{0}^{*} \oplus K\right)$ is a continuous family of $\mathfrak{g}$-superalgebras parametrized by $\mathbb{P}\left(\mathfrak{g}_{0}^{*} \oplus K\right)$ such that the superalgebras corresponding to the points $\xi \in \mathfrak{g}_{0}^{*}$ and $(\xi: 0) \in \mathbb{P}\left(\mathfrak{g}_{0}^{*}\right)$ of the parameter space are $\mathfrak{g}$-equivariantly isomorphic to $U_{\xi}(\mathfrak{g})$ and $S_{\xi}(\mathfrak{g})$, respectively.

Proof. See the proof of [Premet and Skryabin 1999, Proposition 2.2].

Lemma 2.2. Suppose $\pi: A \rightarrow Z$ is a continuous family of $\mathfrak{g}$-superalgebras parametrized by an algebraic variety $Z$. Then, for any positive integer $d$, the set of all points $\zeta \in Z$ such that the corresponding superalgebra $A_{\zeta}$ contains a $\mathfrak{g}$-invariant two-sided ideal of dimension d is closed in $Z$.

Proof. For a superspace $V$, let $\sigma: V \rightarrow V$ be the linear transformation whose action on the homogeneous elements is given by $\sigma(v)=(-1)^{|v|} v$. Then a subspace $W$ of $V$ is graded if and only if $\sigma(W)=(W)$.

Let $\varphi: G_{d}(A) \rightarrow Z$ be the Grassmann bundle of $d$-dimensional subspaces corresponding to the vector bundle $\pi: A \rightarrow Z$. Then the subvariety $G_{d}^{\mathrm{gr}}(A) \subseteq G_{d}(A)$ of graded subspaces of dimension $d$ is closed.

Now follow the proof of [Premet and Skryabin 1999, Lemma 2.3]. 
2g. In the rest of this section, we study the properties of invariant ideals of the reduced symmetric superalgebras. This can be viewed as the super counterpart of [Premet and Skryabin 1999, Section 3]. It turns out that most statements and their proofs therein generalize to the super setup trivially, and so we again omit proofs when they are straightforward generalizations.

Let $\mathfrak{p}$ be a restricted subalgebra of $\mathfrak{g}$, and $\xi \in \mathfrak{g}_{0}^{*}$. For any $U_{\xi}(\mathfrak{p})$-module $V$, the superspace

$$
\widetilde{V}=\operatorname{Hom}_{U_{\xi}(\mathfrak{p})}\left(U_{\xi}(\mathfrak{g}), V\right)
$$

carries a standard $U_{\xi}(\mathfrak{g})$-module structure given by

$$
(x f)(v)=(-1)^{|x|(|f|+|v|)} f(v x),
$$

where $x, v \in U_{\xi}(\mathfrak{g})$, and $f \in \operatorname{Hom}_{U_{\xi}(\mathfrak{p})}\left(U_{\xi}(\mathfrak{g}), V\right)$ are homogeneous elements. This module is called the $U_{\xi}(\mathfrak{g})$-module coinduced from $V$.

Let $A$ be a p-superalgebra. The restricted $\mathfrak{g}$-module $\tilde{A}=\operatorname{Hom}_{U_{0}(\mathfrak{p})}\left(U_{0}(\mathfrak{g}), A\right)$ coinduced from $A$ carries a superalgebra structure such that the $\mathfrak{g}$ acts on $\tilde{A}$ by superderivations. The multiplication in $\tilde{A}$ is given by the formula

$$
(f \cdot g)(u)=\sum_{(u)}(-1)^{|g|\left|u_{(1)}\right|} f\left(u_{(1)}\right) g\left(u_{(2)}\right),
$$

where $f, g \in \tilde{A}$ and $u \in U_{0}(\mathfrak{g})$ are homogenous and $u \mapsto \sum u_{(1)} \otimes u_{(2)}$ is the comultiplication in $U_{0}(\mathfrak{g})$.

2h. Let $\mathfrak{p}$ be a restricted subalgebra of $\mathfrak{g}$. Write $\mathscr{F}(\mathfrak{g}, \mathfrak{p})=\operatorname{Hom}_{U_{0}(\mathfrak{p})}\left(U_{0}(\mathfrak{g}), K\right)$, where $K$ denotes the trivial $U_{0}(\mathfrak{p})$-module.

Lemma 2.3. The superalgebra $\mathscr{F}(\mathfrak{g}, \mathfrak{p})$ is $\mathfrak{g}$-simple and commutative. Moreover, it is isomorphic to a truncated symmetric superalgebra. The unique maximal ideal $\mathfrak{m}(\mathfrak{g}, \mathfrak{p})$ of $\mathscr{F}(\mathfrak{g}, \mathfrak{p})$ consists of all $f \in \mathscr{F}(\mathfrak{g}, \mathfrak{p})$ satisfying $f(1)=0$.

Proof. Let $\left\{x_{1}, \ldots, x_{s}\right\}$ and $\left\{y_{1}, \ldots, y_{t}\right\}$ be elements in $\mathfrak{g}_{\overline{0}}$ and $\mathfrak{g}_{\overline{1}}$, respectively, such that their images form a basis for $\mathfrak{g}_{\overline{0}} / \mathfrak{p}_{\overline{0}}$ and $\mathfrak{g}_{\overline{1}} / \mathfrak{p}_{\overline{1}}$, respectively.

Let

$$
\begin{aligned}
& \Lambda_{\overline{0}}=\left\{\boldsymbol{a}=\left(a_{1}, \ldots, a_{s}\right) \mid 0 \leq a_{i} \leq p-1 \text { are integers }\right\}, \\
& \Lambda_{\overline{1}}=\left\{\boldsymbol{b}=\left(b_{1}, \ldots, b_{r}\right) \mid 1 \leq b_{1}<\cdots<b_{r} \leq t \text { are integers for } 0 \leq r \leq t\right\} .
\end{aligned}
$$

For $\boldsymbol{a}=\left(a_{1}, \ldots, a_{s}\right)$ and $\boldsymbol{a}^{\prime}=\left(a_{1}^{\prime}, \ldots, a_{s}^{\prime}\right)$ in $\Lambda_{\overline{0}}$, let $\boldsymbol{a} !=\prod\left(a_{i} !\right)$. Write $\boldsymbol{a}^{\prime} \leq \boldsymbol{a}$ if $a_{i}^{\prime} \leq a_{i}$ for all $i$. Further put $\left(\begin{array}{c}\boldsymbol{a} \\ \boldsymbol{a}^{\prime}\end{array}\right)=\prod\left(\begin{array}{c}a_{i} \\ a_{i}^{\prime}\end{array}\right)$ when $\mathbf{a}^{\prime} \leq \boldsymbol{a}$. For $\boldsymbol{b}=\left(b_{1}, \ldots, b_{r}\right)$ and $\boldsymbol{b}^{\prime}=\left(b_{1}^{\prime}, \ldots, b_{l}^{\prime}\right)$ in $\Lambda_{\overline{1}}$, write $\boldsymbol{b}^{\prime} \leq \boldsymbol{b}$ if $\left(b_{1}^{\prime}, \ldots, b_{l}^{\prime}\right)$ appears in $\left(b_{1}, \ldots, b_{r}\right)$ as a subsequence. Also, when $\boldsymbol{b}^{\prime} \leq \boldsymbol{b}$, define $\operatorname{sgn}\left(\boldsymbol{b}^{\prime}, \boldsymbol{b}\right)$ to be the sign of the permutation of sequence $\boldsymbol{b}$ given by $\left(\boldsymbol{b}^{\prime}, \boldsymbol{b} \backslash \boldsymbol{b}^{\prime}\right)$, where $\boldsymbol{b} \backslash \boldsymbol{b}^{\prime}$ denotes the subsequence of $\boldsymbol{b}$ formed by removing the subsequence $\boldsymbol{b}^{\prime}$ from $\boldsymbol{b}$. 
For $\boldsymbol{a}=\left(a_{1}, \ldots, a_{s}\right) \in \Lambda_{\overline{0}}$ and $\boldsymbol{b}=\left(b_{1}, \ldots, b_{r}\right) \in \Lambda_{\overline{1}}$, write

$$
e^{(\boldsymbol{a}, \boldsymbol{b})}=x_{1}^{a_{1}} \cdots x_{s}^{a_{s}} y_{b_{1}} \cdots y_{b_{r}} \text {. }
$$

Then $U_{0}(\mathfrak{g})$ is a free $U_{0}(\mathfrak{p})$-module on basis $\left\{e^{(\boldsymbol{a}, \boldsymbol{b})} \mid \boldsymbol{a} \in \Lambda_{\overline{0}}, \boldsymbol{b} \in \Lambda_{\overline{1}}\right\}$ with comultiplication on $e^{(\boldsymbol{a}, \boldsymbol{b})}$ given by

$$
\Delta\left(e^{(\boldsymbol{a}, \boldsymbol{b})}\right)=\sum_{\mathbf{a}^{\prime} \leq \boldsymbol{a} ; \boldsymbol{b}^{\prime} \leq \boldsymbol{b}}\left(\begin{array}{c}
\boldsymbol{a} \\
\boldsymbol{a}^{\prime}
\end{array}\right) \operatorname{sgn}\left(\boldsymbol{b}^{\prime}, \boldsymbol{b}\right) e^{\left(\boldsymbol{a}^{\prime}, \boldsymbol{b}^{\prime}\right)} \otimes e^{\left(\boldsymbol{a}-\boldsymbol{a}^{\prime}, \boldsymbol{b} \backslash \boldsymbol{b}^{\prime}\right)} .
$$

Let $\phi_{i} \in \mathscr{F}(\mathfrak{g}, \mathfrak{p})_{\overline{0}}$ and $\psi_{j} \in \mathscr{F}(\mathfrak{g}, \mathfrak{p})_{\overline{1}}$ be the dual elements of $x_{i}$ for $1 \leq i \leq s$ and $y_{j}$ for $1 \leq j \leq t$ respectively.

Equation (2-1) inductively shows that, for $\boldsymbol{a}, \boldsymbol{a}^{\prime} \in \Lambda_{\overline{0}}$ and $\boldsymbol{b}, \boldsymbol{b}^{\prime} \in \Lambda_{\overline{1}}$,

$$
\phi^{\boldsymbol{a}} \psi^{\boldsymbol{b}}\left(e^{\left(\boldsymbol{a}^{\prime}, \boldsymbol{b}^{\prime}\right)}\right)=\boldsymbol{a} ! \delta_{(\boldsymbol{a}, \boldsymbol{b}),\left(\boldsymbol{a}^{\prime}, \boldsymbol{b}^{\prime}\right)},
$$

where we put $\phi^{\boldsymbol{a}}=\phi_{1}^{a_{1}} \cdots \phi_{s}^{a_{s}}$ and $\psi^{\boldsymbol{b}}=\psi_{b_{1}} \cdots \psi_{b_{r}}$ for $\boldsymbol{a}=\left(a_{1}, \ldots, a_{s}\right)$ and $\boldsymbol{b}=\left(b_{1}, \ldots, b_{r}\right)$.

Then $\mathscr{F}(\mathfrak{g}, \mathfrak{p})$ is an associative superalgebra with unit element and generators $\phi_{1}, \ldots, \phi_{s}$ and $\psi_{1}, \ldots, \psi_{t}$, which satisfy $\phi_{i}^{p}=0$ and $\psi_{j}^{2}=0$ for all $i, j$. Since its dimension is $p^{s} 2^{t}$, there is an isomorphism

$$
K\left[x_{1}, \ldots, x_{s} ; y_{1}, \ldots, y_{t}\right] /\left(x_{1}^{p}, \ldots, x_{s}^{p} ; y_{1}^{2}, \ldots, y_{t}^{2}\right) \cong \mathscr{F}(\mathfrak{g}, \mathfrak{p}) .
$$

To see it is $\mathfrak{g}$-simple, we note inductively from (2-1) that the action of $\mathfrak{g}$ on some of the basis vectors of $\mathscr{F}(\mathfrak{g}, \mathfrak{p})$ is given as follows:

$$
\begin{aligned}
& x_{i} \cdot \phi_{1}^{a_{1}} \cdots \phi_{s}^{a_{s}} \psi^{b} \\
& = \begin{cases}0 & \text { if } a_{i}=0, \\
\lambda \phi_{1}^{a_{1}} \cdots \phi_{i}^{a_{i}-1} \cdots \phi_{s}^{a_{s}} \psi^{b} & \text { if } 2 \leq a_{i} \leq p-1, \\
\mu \phi_{1}^{a_{1}} \cdots \phi_{i-1}^{a_{i-1}} \phi_{i+1}^{a_{i+1}} \cdots \phi_{s}^{a_{s}} \psi^{b}+v \phi_{1}^{a_{1}} \cdots \phi_{i}^{p-1} \cdots \phi_{s}^{a_{s}} \psi^{b} & \text { if } a_{i}=1,\end{cases} \\
& y_{j} \cdot \psi_{j_{1}} \cdots \psi_{j_{r}} \\
& = \begin{cases}0 & \text { if } j \notin\left\{j_{1}, \ldots, j_{r}\right\}, \\
\pm \psi_{j_{1}} \cdots \hat{\psi}_{j} \cdots \psi_{j_{r}} & \text { otherwise, }\end{cases}
\end{aligned}
$$

where $\lambda, \mu$, and $v$ are in $K$ with $\lambda, \mu$ nonzero.

Given any nonzero element in $\mathscr{F}(\mathfrak{g}, \mathfrak{p})$, by applying a suitable sequence of the $x_{i}$ and $y_{j}$, we will eventually arrive at a linear combination of basis vectors $\phi^{\boldsymbol{a}} \psi^{\boldsymbol{b}}$ with nonzero constant term. On the other hand, since $\phi_{i}^{p}=0$ and $\psi_{j}^{2}=0$, every nonzero $\mathfrak{g}$-invariant ideal is nilpotent and contains an element with nonzero constant term. It has to be the whole thing. Hence $\mathscr{F}(\mathfrak{g}, \mathfrak{p})$ is $\mathfrak{g}$-simple. The rest of the statement is clear.

Let $B=B_{0} \oplus B_{\overline{1}}$ be a finite-dimensional unital commutative associative $\mathfrak{g}$ superalgebra. The superalgebra $B$ is said to be $\mathfrak{g}$-simple if it contains no nonzero 
$\mathfrak{g}$-invariant ideals. Arguing as in [Premet and Skryabin 1999, 3.2], we can show that if $B$ is $\mathfrak{g}$-simple, then it is a local superalgebra with the unique maximal ideal $\mathfrak{m}=\mathfrak{m}_{\overline{0}} \oplus B_{\overline{1}}$, where $\mathfrak{m}_{\overline{0}}$ consists of the elements $b \in B_{\overline{0}}$ such that $b^{p}=0$.

Proposition 2.4. Let $B$ be a $\mathfrak{g}$-simple finite-dimensional unital commutative $\mathfrak{g}$ superalgebra. Denote by $\mathfrak{m}$ the maxiaml ideal and by $\mathfrak{p}$ the normalizer of $\mathfrak{m}$ in $\mathfrak{g}$. Then there is a canonical $\mathfrak{g}$-equivariant superalgebra isomorphism $B \cong \mathscr{F}(\mathfrak{g}, \mathfrak{p})$.

Proof. It is similar to the proof of [Premet and Skryabin 1999, Theorem 3.2].

2i. Let $B$ be a commutative $\mathfrak{g}$-superalgebra and $\xi \in \mathfrak{g}_{\overline{0}}^{*}$. By a $\left(B, U_{\xi}(\mathfrak{g})\right)$-module, we mean a $U_{\xi}(\mathfrak{g})$-module that is also a module over superalgebra $B$ such that the module structure map $B \otimes M \rightarrow M$ is a $\mathfrak{g}$-module homomorphism. A $(B, \mathfrak{g})$ superalgebra is a $K$-superalgebra $C$, which is simultaneously a $B$-superalgebra and $\mathfrak{g}$-superalgebra and a $\left(B, U_{0}(\mathfrak{g})\right)$-module.

Now let $B=\mathscr{F}(\mathfrak{g}, \mathfrak{p})$. For any $U_{\xi}(\mathfrak{p})$-module $V$, the coinduced $U_{\xi}(\mathfrak{g})$-module $\widetilde{V}$ carries a canonical $\left(\mathscr{F}(\mathfrak{g}, \mathfrak{p}), U_{\xi}(\mathfrak{g})\right)$-module structure given by $(f \cdot \psi)(u)=$ $\sum_{(u)}(-1)^{|\psi|\left|u_{(1)}\right|} f\left(u_{(1)}\right) \psi\left(u_{(2)}\right)$, where $f \in \mathscr{F}(\mathfrak{g}, \mathfrak{p}), \quad \psi \in \widetilde{V}$, and $u \in U_{\xi}(\mathfrak{g})$ are homogeneous.

If $A$ is a $\mathfrak{p}$-superalgebra, the $\left(\mathscr{F}(\mathfrak{g}, \mathfrak{p}), U_{0}(\mathfrak{g})\right)$-module $\tilde{A}=\operatorname{Hom}_{U_{0}(\mathfrak{p})}\left(U_{0}(\mathfrak{g}), A\right)$ has a $\mathfrak{g}$-invariant multiplication and is $\mathscr{F}(\mathfrak{g}, \mathfrak{p})$-bilinear as well. Therefore, $\tilde{A}$ is an $(\mathscr{F}(\mathfrak{g}, \mathfrak{p}), \mathfrak{g})$-superalgebra.

Proposition 2.5. Let $M$ be an $\left(\mathscr{F}(\mathfrak{g}, \mathfrak{p}), U_{\xi}(\mathfrak{g})\right)$-module and $C$ an $(\mathscr{F}(\mathfrak{g}, \mathfrak{p}), \mathfrak{g})$ superalgebra. Then

(i) $M \cong \operatorname{Hom}_{U_{\xi}(\mathfrak{p})}\left(U_{\xi}(\mathfrak{g}), M / \mathfrak{m}(\mathfrak{g}, \mathfrak{p}) M\right)$ as $\left(\mathscr{F}(\mathfrak{g}, \mathfrak{p}), U_{\xi}(\mathfrak{g})\right)$-module, and

(ii) $C \cong \operatorname{Hom}_{U_{0}(\mathfrak{p})}\left(U_{0}(\mathfrak{g}), C / \mathfrak{m}(\mathfrak{g}, \mathfrak{p}) C\right)$ as $(\mathscr{F}(\mathfrak{g}, \mathfrak{p})$, $\mathfrak{g})$-superalgebras.

Proof. It is similar to the proof of [Premet and Skryabin 1999, Theorem 3.3].

2j. Let $\xi \in \mathfrak{g}_{0}^{*}$. Recall that the centralizer of $\xi$ in $\mathfrak{g}$ is denoted by $\mathfrak{g}_{\xi}$, which is a restricted Lie subalgebra. Put $d_{0} \mid d_{1}=\underline{\operatorname{codim}} \mathfrak{g}_{\xi}$.

Proposition 2.6. Let $\xi \in \mathfrak{g}_{\overline{0}}^{*}$ and $d_{0} \mid d_{1}=\underline{\operatorname{codim}} \mathfrak{g}_{\xi}$. Then each $\mathfrak{g}$-invariant ideal of $S_{\xi}(\mathfrak{g})$ has codimension divisible by $p^{d_{0}} 2^{d_{1}}$. Among them there is a unique maximal one of codimension $p^{d_{0}} 2^{d_{1}}$.

Proof. The proof, which uses Propositions 2.4 and 2.5(i), is similar to proof of [Premet and Skryabin 1999, Theorem 3.4].

Remark 2.7. Let $\mathscr{L}$ be an $n$-dimensional restricted Lie algebra, and let $r$ be the minimal dimension of the centralizers of all $\chi \in \mathscr{L}^{*}$. Kac and Weisfeiler have conjectured that the maximal dimension $M(\mathscr{L})$ of simple $\mathscr{L}$-modules is $p^{(n-r) / 2}$. Like [Premet and Skryabin 1999], we refer to this as the KW1 conjecture. 
Premet and Skryabin [1999] showed that

(†) the set of $\chi \in \mathscr{L}^{*}$ such that all the simple modules of $U_{\chi}(\mathscr{L})$ have the maximal dimension $M(\mathscr{L})$ is nonempty and Zariski open in $\mathscr{L}^{*}$.

Using deformation arguments, they then showed that

( ) if there is $\chi \in \mathscr{L}^{*}$ whose centralizer is a toral subalgebra of $\mathscr{L}$, then there is a nonempty and Zariski open subset $W$ of $\mathscr{L}^{*}$ such that $\xi \in W$ implies that all simple $U_{\xi}(\mathscr{L})$-modules have dimension $p^{(n-r) / 2}$.

This, together with $(\dagger)$, confirms the KW1 conjecture for such $\mathscr{L}$.

Using the arguments in this section, we can establish the corresponding statement of $(\ddagger)$ in the superalgebra setting. However, it is not clear how to generalize $(\dagger)$ to a general restricted Lie superalgebra. This is mainly because the universal enveloping superalgebra is in general not a prime ring (see [Bell 1990] for a counterexample over the complex numbers), which is crucial in the proof of $(\dagger)$ in [Premet and Skryabin 1999].

\section{Proof of super KW property for basic classical Lie superalgebras}

3a. In this subsection we first recall some basic facts about the method of associated cones, following [Premet and Skryabin 1999, Section 5.1].

Let $V$ be a finite-dimensional vector space over $K$. For an ideal $I$ of the symmetric algebra $S\left(V^{*}\right)$, let gr $I$ denote the homogeneous ideal of $S\left(V^{*}\right)$ with the property that $g \in \operatorname{gr} I \cap S^{r}\left(V^{*}\right)$ if and only if there is a $\tilde{g} \in I$ such that $\tilde{g}-g \in \bigoplus_{j<r} S^{j}\left(V^{*}\right)$. Identify $S\left(V^{*}\right)$ with the algebra of polynomial functions on $V$. Associate to a subset $X \subseteq V$ the ideal

$$
I_{X}=\left\{g \in S\left(V^{*}\right) \mid g(X)=0\right\} .
$$

The set $\mathbb{K} X:=\left\{v \in V \mid f(v)=0\right.$ for all $\left.f \in \operatorname{gr} I_{X}\right\}$. is called the cone associated with $X$. It is a Zariski closed conical subset of $V$. We identify $V$ with the subset of $\mathbb{P}(V \oplus K)$ consisting of all points $(v: 1)$ with $v \in V$, and identify $\mathbb{P}(V)$ with the subset of $\mathbb{P}(V \oplus K)$ consisting of all points $(v: 0)$ with $v \in V \backslash\{0\}$. Let $\bar{X}^{P}$ and $\bar{X}$ denote the Zariski closure of $X$ in $\mathbb{P}(V \oplus K)$ and in $V$, respectively. It is easy to prove that

$$
\bar{X}^{P} \cap V=\bar{X} \text { and } \bar{X}^{P} \cap \mathbb{P}(V)=\mathbb{P}(\mathbb{K} X),
$$

where $\mathbb{P}(\mathbb{K} X) \subseteq \mathbb{P}(V)$ denotes the projectivization of the conical subset $\mathbb{R} X$.

3b. Let $\mathfrak{g}$ be a restricted Lie superalgebra. For a pair of nonnegative integers $\left(d_{0} \mid d_{1}\right)$ with $d_{0}$ even, let $\mathscr{X}_{d_{0}, d_{1}}$ denote the set of all $\xi \in \mathfrak{g}_{0}^{*}$ such that the algebra $U_{\xi}(\mathfrak{g})$ has a module of finite dimension not divisible by $p^{d_{0} / 2} 2^{\left\lfloor d_{1} / 2\right\rfloor}$, and let 
$\mathscr{X}_{d_{0}, d_{1}}^{\prime} \subseteq \mathbb{P}\left(\mathfrak{g}_{0}^{*} \oplus K\right)$ be the subset of all points $(\xi: \lambda)$ such that $U_{\xi, \lambda}(\mathfrak{g})$ has a $\mathfrak{g}$-invariant ideal of codimension not divisible by $p^{d_{0}} 2^{d_{1}}$. Set

$$
\mathscr{Y}_{d_{0}, d_{1}}=\left\{\xi \in \mathfrak{g}_{\overline{0}}^{*} \mid \operatorname{codim}_{\mathfrak{g}_{\overline{0}}} \mathfrak{g}_{\xi, \overline{0}}<d_{0} \text { or } \operatorname{codim}_{\mathfrak{g}_{\overline{1}}} \mathfrak{g}_{\xi, \overline{1}}<d_{1}\right\} .
$$

Note that $\mathscr{X}_{d_{0}, 2 k+1}=\mathscr{X}_{d_{0}, 2 k+2}$, but this is not the case for $\mathscr{X}_{d_{0}, d_{1}}^{\prime}$ and $\mathscr{Y}_{d_{0}, d_{1}}$.

By Lemma 2.2, $\mathscr{X}_{d_{0}, d_{1}}^{\prime}$ is closed. The set $\mathscr{Y}_{d_{0}, d_{1}}$ is obviously conical, and let $\mathbb{P}\left(\mathscr{Y}_{d_{0}, d_{1}}\right) \subseteq \mathbb{P}\left(\mathfrak{g}_{0}^{*}\right)$ be its projectivization. By Proposition 2.6, $\eta \in \mathfrak{g}_{0}^{*}$ lies in $\mathscr{Y}_{d_{0}, d_{1}}$ if and only if $S_{\eta}(\mathfrak{g})$ has a $\mathfrak{g}$-invariant ideal with codimension not divisible by $p^{d_{0}} 2^{d_{1}}$. Therefore

$$
\mathscr{X}_{d_{0}, d_{1}}^{\prime} \cap \mathbb{P}\left(\mathfrak{g}_{0}^{*}\right)=\mathbb{P}\left(\mathscr{Y}_{d_{0}, d_{1}}\right) .
$$

Hence $\mathbb{P}\left(\mathscr{Y}_{d_{0}, d_{1}}\right)$ is closed in $\mathbb{P}\left(\mathfrak{g}_{\overline{0}}^{*}\right)$, and so $\mathscr{Y}_{d_{0}, d_{1}}$ is Zariski closed in $\mathfrak{g}_{0}^{*}$.

Proposition 3.1. $\mathbb{K} \mathscr{X}_{d_{0}, d_{1}} \subseteq \mathscr{Y}_{d_{0}, d_{1}}$ for any pair of nonnegative integers $\left(d_{0} \mid d_{1}\right)$ with $d_{0}$ even.

Proof. We claim that $\mathscr{X}_{d_{0}, d_{1}} \subseteq \mathscr{X}_{d_{0}, d_{1}}^{\prime} \cap \mathfrak{g}_{0}^{*}$. Indeed, suppose $\xi \in \mathfrak{g}_{0}^{*} \backslash\left(\mathscr{X}_{d_{0}, d_{1}}^{\prime} \cap \mathfrak{g}_{0}^{*}\right)$. Then since all the two-sided ideals of $U_{\xi}(\mathfrak{g})$ are $\mathfrak{g}$-invariant, the codimension of each two-sided ideal of $U_{\xi}(\mathfrak{g})$ is divisible by $p^{d_{0}} 2^{d_{1}}$. Let $V$ be a simple module of $U_{\xi}(\mathfrak{g})$ and let $J=\operatorname{Ann}_{U_{\xi}(\mathfrak{g})} V$ be its annihilator in $U_{\xi}(\mathfrak{g})$. Then by [Liu et al. 1991, Section 2], $U_{\xi}(\mathfrak{g}) / J$ is a simple superalgebra over $K$ with the unique simple module $V$ since $K$ is algebraically closed. Then either $U_{\xi}(\mathfrak{g}) / J$ is of type $M$ with dimension $a^{2}$ for some natural number $a$, or it is of type $Q$ with dimension $2 b^{2}$ for some natural number $b$. In the first case, the dimension of $V$ is $a$. Then since $p^{d_{0}} 2^{d_{1}}$ divides $a^{2}=\operatorname{dim} U_{\xi}(\mathfrak{g}) / J$, we will have $p^{d_{0} / 2} 2^{\left\lfloor d_{1} / 2\right\rfloor} \operatorname{divides} a$. In the second case, the dimension of $V$ is $2 b$. Since $p^{d_{0}} 2^{d_{1}} \operatorname{divides} 2 b^{2}=\operatorname{dim} U_{\xi}(\mathfrak{g}) / J$, $p^{d_{0} / 2} 2^{\left\lceil d_{1} / 2\right\rceil}$ divides $b$. In either case, the $U_{\xi}(\mathfrak{g})$-module $V$ has dimension divisible by $p^{d_{0} / 2} 2^{\left\lfloor d_{1} / 2\right\rfloor}$, which implies $\xi \notin \mathscr{X}_{d_{0}, d_{1}}$. The claim is proved.

The claim implies that $\overline{\mathscr{X}} \mathbb{d}_{0}, d_{1} \subseteq \mathscr{X}_{d_{0}, d_{1}}^{\prime}$, since $\mathscr{X}_{d_{0}, d_{1}}^{\prime}$ is closed by Lemma 2.2. Then we have $\overline{\mathscr{X}}_{d_{0}, d_{1}}^{P} \cap \mathbb{P}\left(\mathfrak{g}_{0}^{*}\right) \subseteq \mathscr{X}_{d_{0}, d_{1}}^{\prime} \cap \mathbb{P}\left(\mathfrak{g}_{0}^{*}\right)$, which means $\mathbb{P}\left(\mathbb{K} \mathscr{X}_{d_{0}, d_{1}}\right) \subseteq \mathbb{P}\left(\mathscr{Y}_{d_{0}, d_{1}}\right)$ by (3-1) and (3-2). But since both $\mathbb{K} \mathscr{X}_{d_{0}, d_{1}}$ and $\mathscr{Y}_{d_{0}, d_{1}}$ are conical, we deduce that $\mathbb{K} \mathscr{X}_{d_{0}, d_{1}} \subseteq \mathscr{Y}_{d_{0}, d_{1}}$, as desired.

3c. Let $G\left(\mathfrak{g}_{\overline{0}}\right)$ denote the group of all automorphisms of $\mathfrak{g}_{\overline{0}}$ preserving the $[p]$-th power map, that is, automorphisms $g$ satisfying $g\left(x^{[p]}\right)=g(x)^{[p]}$ for all $x \in \mathfrak{g}_{\overline{0}}$. Let $\Omega(\eta)$ denote the $G\left(\mathfrak{g}_{0}\right)$-orbit of $\eta \in \mathfrak{g}_{0}^{*}$.

For $\chi \in \mathfrak{g}_{\overline{0}}^{*}$, define $l_{0}(\chi)=\min _{\xi \in \mathbb{K} \Omega \Omega(\chi)} \operatorname{dim} \mathfrak{g}_{\xi, \overline{0}}$ and $l_{1}(\chi)=\min _{\xi \in \mathbb{K} \Omega \Omega(\chi)} \operatorname{dim} \mathfrak{g}_{\xi, \overline{1}}$.

Theorem 3.2. Let $\mathfrak{g}$ be an $\left(n_{0} \mid n_{1}\right)$-dimensional restricted Lie superalgebra, and let $\chi \in \mathfrak{g}_{\overline{0}}^{*}$. Write $l_{i}=l_{i}(\chi)$ for $i \in \mathbb{Z}_{2}$, and $d_{0} \mid d_{1}=\underline{\operatorname{codim}}_{\mathfrak{g}} \mathfrak{g}_{\chi}$.

(i) The dimension of each finite-dimensional $U_{\chi}(\mathfrak{g})$-module is an integer multiple of $p^{\left(n_{0}-l_{0}\right) / 2} 2^{\left\lfloor\left(n_{1}-l_{1}\right) / 2\right\rfloor}$. 
(ii) If all nonzero scalar multiples of $\chi$ are $G\left(\mathfrak{g}_{0}\right)$-conjugate, then the dimensions of all finite-dimensional $U_{\chi}(\mathfrak{g})$-modules are divisible by $p^{d_{0} / 2} 2^{\left\lfloor d_{1} / 2\right\rfloor}$, that is, the super $K W$ conjecture holds for the algebra $U_{\chi}(\mathfrak{g})$.

Proof. To prove part (i), we treat the $p$ - and 2-divisibility separately. Suppose that $U_{\xi}(\mathfrak{g})$ has a finite-dimensional module $V$ such that $\operatorname{dim} V$ is not divisible by $2^{\left\lfloor\left(n_{1}-l_{1}\right) / 2\right\rfloor}$. Then $\chi \in \mathscr{X}_{0, n_{1}-l_{1}}$ by the definition of $\mathscr{X}_{0, n_{1}-l_{1}}$. (Note that in addition, $\chi \in \mathscr{X}_{0, n_{1}-l_{1}+1}$ when $n_{1}-l_{1}$ is odd, while $\chi \in \mathscr{X}_{0, n_{1}-l_{1}-1}$ when $n_{1}-l_{1}$ is even. But we do not need this.) For any $g \in G\left(\mathfrak{g}_{0}\right)$, the algebras $U_{g(\chi)}(\mathfrak{g})$ and $U_{\chi}(\mathfrak{g})$ are isomorphic. It follows that $\Omega(\chi) \subseteq \mathscr{X}_{0, n_{1}-l_{1}}$. But then $\mathbb{K} \Omega(\chi) \subseteq \mathbb{K} \mathscr{X}_{0, n_{1}-l_{1}}$. Since $\mathbb{K} \mathscr{Q}_{0, n_{1}-l_{1}} \subseteq \mathscr{Y}_{0, n_{1}-l_{1}}$ by Proposition 3.1, we have $\operatorname{codim}_{\mathfrak{g}_{\overline{1}}} \mathfrak{g}_{\xi, \overline{1}}<n_{1}-l_{1}$ for any $\xi \in \mathbb{K} \Omega(\chi)$, which contradicts the choice of $l_{1}$.

The $p$-divisibility can be proved similarly.

For part (ii), note first that $(\chi: 0) \in \overline{K^{\times} \chi} P$. Since by assumption $K^{\times} \chi$ is contained in a single $G\left(\mathfrak{g}_{\overline{0}}^{*}\right)$-orbit, we have by (3-1)

$$
(\chi: 0) \in{\overline{K^{\times}}}^{P} \cap \mathbb{P}\left(\mathfrak{g}_{0}^{*}\right) \subseteq \overline{\Omega(\chi)}^{P} \cap \mathbb{P}\left(\mathfrak{g}_{0}^{*}\right)=\mathbb{P}(\mathbb{K} \Omega(\chi)) .
$$

Thus $\chi \in \mathbb{K} \Omega(\chi)$, and as a result, $l_{i} \leq n_{i}-d_{i}$ for $i \in \mathbb{Z}_{2}$. From here, (ii) follows from (i).

3d. Now let $\mathfrak{g}$ be one of the basic classical Lie superalgebras as in Section $2 \mathrm{~d}$. Recall that the even subalgebra $\mathfrak{g}_{\overline{0}}$ is the Lie algebra of a reductive group $G_{\overline{0}}$, and that $\mathfrak{g}$ admits an even nondegenerate $G_{\overline{0}}$-invariant bilinear form. Given the bilinear form, we can speak of nilpotent $p$-characters, that is, those that correspond to nilpotent elements in $\mathfrak{g}_{0}$ under the isomorphism $\mathfrak{g}_{\overline{0}} \cong \mathfrak{g}_{0}^{*}$ induced by the bilinear form.

We are now ready to reprove [Wang and Zhao 2009a, Theorem. 4.3].

Theorem 3.3. Let $\mathfrak{g}$ be as in Section $2 d$, and let $\chi \in \mathfrak{g}_{0}^{*}$ be nilpotent. Write $d_{0} \mid d_{1}=\underline{\operatorname{codim}} \mathfrak{g}_{\chi}$. Then the dimension of every finite-dimensional $U_{\chi}(\mathfrak{g})$-module $V$ is divisible by $p^{d_{0} / 2} 2^{\left\lfloor d_{1} / 2\right\rfloor}$.

Proof. By [Jantzen 2004, Theorem 2.8.1], $G_{\overline{0}}$ has finitely many orbits in $\mathfrak{g}_{\overline{0}}$. Thus $G_{\overline{0}}$ has finitely many coadjoint orbits in $\mathfrak{g}_{\overline{0}}^{*}$ via the $G_{\overline{0}}$-equivariant isomorphism $\mathfrak{g}_{0} \cong \mathfrak{g}_{\overline{0}}^{*}$. If $\chi \in \mathfrak{g}_{\overline{0}}^{*}$ is nilpotent, so is $K^{\times} \chi$. Then by [Jantzen 2004, Lemma 2.10], $K^{\times} \chi$ is contained in the $G_{\overline{0}}$-orbit of $\chi$. Now since $\operatorname{Ad}_{\overline{0}} \subseteq G\left(\mathfrak{g}_{0}\right)$, we have $K^{\times} \chi \subseteq G_{0} \cdot \chi \subseteq \Omega(\chi)$. Hence by Theorem 3.2(ii), the dimension of every finitedimensional $U_{\chi}(\mathfrak{g})$-module $V$ is divisible by $p^{d_{0} / 2} 2^{\left\lfloor d_{1} / 2\right\rfloor}$, that is, the super $\mathrm{KW}$ conjecture holds for $U_{\chi}(\mathfrak{g})$.

Remark 3.4. In a fashion similar to Theorem 3.3, we can use Theorem 3.2 to give an alternative proof of super $\mathrm{KW}$ conjecture for the queer Lie superalgebra with nilpotent $p$-characters [Wang and Zhao 2009b, Theorem 4.4]. 
Now together with [Wang and Zhao 2009a, Remarks 2.5 and 4.6, Theorem 5.2], we have strengthened the super KW property for basic classical Lie superalgebras as follows. We remark that [Wang and Zhao 2009a, Theorem 5.2] remains valid for basic classical Lie superalgebras with assumption on $p$ as in Section $2 \mathrm{~d}$.

Theorem 3.5 (super Kac-Weisfeiler conjecture). Let $\mathfrak{g}$ be a basic classical Lie superalgebra as in Section $2 d$, and let $\chi \in \mathfrak{g}_{\overline{0}}^{*}$. Let $d_{0} \mid d_{1}=\underline{\operatorname{codim}} \mathfrak{g}_{\chi}$. Then the dimension of every $U_{\chi}(\mathfrak{g})$-module $M$ is divisible by $p^{d_{0} / 2} 2^{\left\lfloor d_{1} / 2\right\rfloor}$.

\section{Semisimple $p$-characters for basic classical Lie superalgebras}

Now we turn our attention to basic classical Lie superalgebras $\mathfrak{g}$ (see Section 2d) with a semisimple $p$-character $\chi \in \mathfrak{g}_{0}^{*}$, defined below. Our purpose is to give a semisimplicity criterion for the reduced enveloping superalgebra $U_{\chi}(\mathfrak{g})$.

Let $\mathfrak{g}$ be one of the basic classical Lie superalgebras of Section $2 d$. A Cartan subalgebra $\mathfrak{h}$ of $\mathfrak{g}$ or of $\mathfrak{g}_{\overline{0}}$ defines the set of roots $\Delta=\Delta_{\overline{0}} \cup \Delta_{\overline{1}}$, the union of even roots $\Delta_{\overline{0}}$ and odd roots $\Delta_{\overline{1}}$. Let $W$ be the Weyl group of $\mathfrak{g}_{\overline{0}}$. The $G_{\overline{0}}$-invariant bilinear form on $\mathfrak{g}$ induces a $W$-invariant bilinear form $(\cdot, \cdot)$ on $\mathfrak{h}^{*}$. Put

$$
\begin{aligned}
& \bar{\Delta}_{\overline{0}}=\left\{\alpha \in \Delta_{\overline{0}} \mid \frac{1}{2} \alpha \notin \Delta_{\overline{1}}\right\}, \\
& \bar{\Delta}_{\overline{1}}=\left\{\alpha \in \Delta_{\overline{1}} \mid 2 \alpha \notin \Delta_{\overline{0}}\right\}=\left\{\alpha \in \Delta_{\overline{1}} \mid(\alpha, \alpha)=0\right\} .
\end{aligned}
$$

For $\alpha \in \Delta$, let $H_{\alpha}$ and $X_{\alpha}$ be a choice of coroot and root vectors, respectively.

Let $\chi \in \mathfrak{g}_{0}^{*}$ be a $p$-character satisfying $\chi\left(X_{\alpha}\right)=0$ for all $\alpha \in \Delta_{\overline{0}}$. A $p$-character that is $G_{0}$-conjugate to one of such $\chi$ is called semisimple.

4a. Fix an arbitrary set of simple roots $\Pi$ of $\Delta$. It determines a set of positive roots $\Pi_{\Delta^{+}}$. Denote by $\Pi_{\Delta_{\overline{0}}^{+}}, \Pi_{\Delta_{\overline{1}}^{+}}, \Pi_{\bar{\Delta}_{\overline{0}}^{+}}$, and ${ }^{\Pi_{\Delta_{\overline{1}}^{+}}^{+}}$the subsets of positive roots in the sets $\Delta_{\overline{0}}, \Delta_{\overline{1}}$, and so on. Let

$$
\mathfrak{g}={ }^{\Pi_{\mathfrak{n}}}{ }^{-} \oplus \mathfrak{h} \oplus \Pi_{\mathfrak{n}^{+}}
$$

be the corresponding triangular decomposition. Put $\Pi_{\mathfrak{b}}=\mathfrak{h} \oplus \Pi_{\mathfrak{n}^{+}}$. Let $\Pi_{\rho}=$ ${ }^{\Pi} \rho_{\overline{0}}-{ }^{\Pi} \rho_{\overline{1}}$, where ${ }^{\Pi} \rho_{\overline{0}}$ and ${ }^{\Pi} \rho_{\overline{1}}$ is the half sum of the positive even and odd roots, respectively.

For $\lambda \in \Lambda_{\chi}:=\left\{\lambda \in \mathfrak{h}^{*} \mid \lambda(h)^{p}-\lambda\left(h^{[p]}\right)=\chi(h)^{p}\right.$ for all $\left.h \in \mathfrak{h}\right\}$, the baby Verma module $Z_{\chi}^{\Pi}(\lambda)$ is defined to be $Z_{\chi}^{\Pi}(\lambda):=U_{\chi}(\mathfrak{g}) \otimes_{U_{\chi}\left(\Pi_{\mathfrak{b}}\right)} K_{\lambda}$, where $K_{\lambda}$ is the onedimensional $U_{\chi}\left(\Pi_{\mathfrak{b}}\right)$-module upon which $\mathfrak{h}$ acts via multiplication by $\lambda$ and $\Pi_{\mathfrak{n}}{ }^{+}$ acts as zero. Write $v_{\lambda}=1 \otimes 1_{\lambda}$ in $Z_{\chi}^{\Pi}(\lambda)$.

Index roots in ${ }^{\Pi} \Delta^{+}$by $\{1,2, \ldots, N=|\Delta| / 2\}$ in a way that is compatible with heights of roots, that is, the shorter the root is in height the smaller it is indexed. For $\alpha \in{ }^{\Pi} \Delta^{+}$, put

$$
m_{\alpha}= \begin{cases}p-1 & \text { if } \alpha \in{ }^{\Pi} \Delta_{\overline{0}}^{+}, \\ 1 & \text { if } \alpha \in{ }^{\Pi} \Delta_{\overline{1}}^{+} .\end{cases}
$$


Lemma 4.1. The vector $X_{-\alpha_{1}}^{m_{\alpha_{1}}} \cdots X_{-\alpha_{N}}^{m_{\alpha_{N}}} v_{\lambda}$ is an element of any nonzero submodule $S$ of $Z_{\chi}^{\Pi}(\lambda)$.

Proof. The proof is similar to the proof of [Rudakov 1970, Proposition 4].

Lemma 4.2. In $U(\mathfrak{g})$, we have

$$
X_{\alpha_{1}}^{m_{\alpha_{1}}} \cdots X_{\alpha_{N}}^{m_{\alpha_{N}}} X_{-\alpha_{1}}^{m_{\alpha_{1}}} \cdots X_{-\alpha_{N}}^{m_{\alpha_{N}}}-{ }^{\Pi} \Phi \in U(\mathfrak{g})^{\Pi_{\mathfrak{n}}} \mathfrak{n}^{+},
$$

where $\Pi \Phi$ is a polynomial in $\left\{H_{\alpha} \mid \alpha \in \Pi\right\}$ of degree $\left(\frac{1}{2}(p-1)\left|\Delta_{\overline{0}}\right|+\frac{1}{2}\left|\Delta_{\overline{1}}\right|\right)$.

Proof. The proof is similar to the proof of [Rudakov 1970, Proposition 5].

Proposition 4.3. The baby Verma module $Z_{\chi}^{\Pi}(\lambda)$ is irreducible if and only if $\Pi_{\Phi}(\lambda) \neq 0$ for $\lambda \in \Lambda_{\chi}$.

Proof. This follows readily from Lemmas 4.1 and 4.2.

Put ${ }^{\Pi} \Phi^{\prime}(\lambda)={ }^{\Pi} \Phi\left(\lambda-{ }^{\Pi} \rho\right)$ for $\lambda \in \mathfrak{h}^{*}$.

4b. Retain the notation from the previous subsection. A simple root $\delta \in \Pi$ is one of the following three types:

(i) $\delta \in \bar{\Delta}_{\overline{0}}$,

(ii) $\delta \in \bar{\Delta}_{\overline{1}}$,

(iii) $\delta \in \Delta_{\overline{1}} \backslash \bar{\Delta}_{\overline{1}}$ with $2 \delta \in \Delta_{\overline{0}} \backslash \bar{\Delta}_{\overline{0}}$.

For such a $\delta$, we shall write

$$
\delta^{*}= \begin{cases}\delta & \text { in case (i) and (ii) } \\ \{\delta, 2 \delta\} & \text { in case (iii) }\end{cases}
$$

Let $r_{\delta}$ be the (even or odd) reflection associated to $\delta$. When $\delta$ is of type (i), $r_{\delta}$ is just the even reflection in $\mathfrak{h}^{*}$ defined by

$$
r_{\delta}(\lambda)=\lambda-\frac{2(\delta, \lambda)}{(\delta, \delta)} \delta \quad \text { for } \lambda \in \mathfrak{h}^{*}
$$

When $\delta$ is of type (iii), $r_{\delta}$ is by definition the even reflection $r_{2 \delta}$, which is also given by formula (4-1). When $\delta$ is of type (ii), $r_{\delta}$ is given by

$$
r_{\delta}(\beta)= \begin{cases}-\delta & \text { if } \beta=\delta, \\ \beta+\delta & \text { if }(\delta, \beta) \neq 0, \\ \beta & \text { if } \beta \neq \delta \text { and }(\delta, \beta)=0 .\end{cases}
$$

It is known (see for example [Serganova 2008]) that $r_{\delta} \Pi$ is the set of simple roots of the positive system ${ }^{r_{\delta} \Pi_{\Delta}} \Delta^{+}:=r_{\delta}\left({ }^{\Pi} \Delta^{+}\right)$, which satisfies $-\delta^{*} \in{ }^{r_{\delta} \Pi} \Delta^{+}$and $r_{\delta} \Pi_{\Delta^{+}} \cap \Pi_{\Delta^{+}}={ }^{\Pi} \Delta^{+} \backslash \delta^{*}$.

By going through the argument of the previous subsection, we know that there is a polynomial ${ }^{r_{\delta} \Pi} \Phi$ on $\mathfrak{h}^{*}$ of degree $\left(\frac{1}{2}(p-1)\left|\Delta_{0}\right|+\frac{1}{2}\left|\Delta_{\overline{1}}\right|\right)$ satisfying ${ }^{r_{\delta} \Pi} \Phi(\lambda) \neq 0$ 
if and only if the baby Verma module $Z_{\chi}^{r_{\delta} \Pi}(\lambda)$ associated to the positive system $r_{\delta} \Pi_{\Delta^{+}}$is irreducible for any $\lambda \in \Lambda_{\chi}$.

For two polynomials $f_{1}$ and $f_{2}$, write $f_{1} \sim f_{2}$ if $f_{1}=c f_{2}$ for some $c \in K^{\times}$.

Lemma 4.4. We have ${ }^{r_{\delta} \Pi} \Phi^{\prime} \sim \Pi^{\prime} \Phi^{\prime}$ for a simple root $\delta \in \Pi$.

Proof. Let us prove it for $\delta$ of type (iii); the other two cases can be proved similarly. First we observe that the vector $X_{-\delta} X_{-2 \delta}^{p-1} v_{\lambda}$ in $Z_{\chi}^{\Pi}(\lambda)$ is annihilated by any root vector $X_{\alpha}$ for $\alpha \in{ }^{r_{\delta} \Pi} \Delta^{+}$. It follows that there is a nontrivial $U(\mathfrak{g})$-module homomorphism $Z_{\chi}^{r_{\delta} \Pi}(\lambda+\delta) \rightarrow Z_{\chi}^{\Pi}(\lambda)$. Since the two baby Verma modules have the same dimension, the reducibility of $Z_{\chi}^{r_{\delta} \Pi}(\lambda+\delta)$ will imply that of $Z_{\chi}^{\Pi}(\lambda)$. By Proposition 4.3, we have ${ }^{r_{\delta} \Pi} \Phi(\lambda+\delta)$ divides ${ }^{\Pi} \Phi(\lambda)$, and so ${ }^{r_{\delta} \Pi} \Phi(\lambda+\delta) \sim{ }^{\Pi} \Phi(\lambda)$. Hence ${ }^{r_{\delta} \Pi} \Phi^{\prime}(\lambda) \sim \Pi_{\Phi^{\prime}}(\lambda)$ since ${ }^{r_{\delta} \Pi} \rho=\Pi_{\rho}-\delta$.

When $\delta$ is of type (i), then as in the classical case, the vector $X_{-\delta}^{p-1} v_{\lambda}$ in $Z_{\chi}^{\Pi}(\lambda)$ is a singular vector for the positive system ${ }^{r} \Pi \Delta^{+}$. We then can argue the same way as for the $\delta$ of type (iii).

When $\delta$ is of type (ii), we only need to observe that the vector $X_{-\delta} v_{\lambda}$ in $Z_{\chi}^{\Pi}(\lambda)$ is a singular vector for the positive system ${ }^{r_{\delta}} \Pi_{\Delta}{ }^{+}$. The rest of the argument is the same as for the $\delta$ of type (iii).

Since by applying (even and odd) simple reflections, we can obtain any set $\tilde{\Pi}$ of simple roots from a given set $\Pi$ of simple roots, we conclude by Lemma 4.4 that the polynomial ${ }^{\tilde{\Pi}} \Phi^{\prime}$ does not depend on the choice of $\tilde{\Pi}$ up to equivalence by $\sim$. Thus we can suppress the left superscript $\tilde{\Pi}$ of ${ }^{\tilde{\Pi}} \Phi^{\prime}$ and write $\Phi^{\prime}$ instead.

Proposition 4.5. We have $\Phi^{\prime}(\lambda) \sim \prod_{\alpha \in \Pi_{\Delta_{\overline{0}}^{+}}}\left((\lambda \mid \alpha)^{p-1}-1\right) \cdot \prod_{\beta \in \Pi_{\Delta_{\overline{1}}^{+}}}(\lambda \mid \beta)$ for any set of simple roots $\Pi$.

A different choice of simple roots in Proposition 4.5 will only lead to a different sign in the product on the right hand side.

Proof. If $\Delta_{\overline{1}} \backslash \bar{\Delta}_{\overline{1}} \neq \varnothing$, then any $\delta \in \Delta_{\overline{1}} \backslash \bar{\Delta}_{\overline{1}}$ appears as a simple root in some set $\tilde{\Pi}$ of simple roots. The root vector $X_{\delta}$ generates an embedded osp $(1 \mid 2)$ in $\mathfrak{g}$. Consider the minimal parabolic subalgebra $\mathfrak{p}=\mathfrak{o s p}(1 \mid 2)+\tilde{\Pi}_{\mathfrak{b}}$ and the induced module

$$
Z_{\chi}^{\mathfrak{p}}(\lambda)=U_{\chi}(\mathfrak{p}) \otimes_{U_{\chi}\left(\tilde{\Pi}_{\mathfrak{b}}\right)} K_{\lambda} .
$$

The $U_{\chi}(\mathfrak{p})$-module $Z_{\chi}^{\mathfrak{p}}(\lambda)$ is merely the baby Verma module $Z_{\chi}^{\mathfrak{o s p}(1 \mid 2)}(\lambda)$ of the embedded $\mathfrak{o s p}(1 \mid 2)$ upon which $\mathfrak{h}$ acts as weight multiplication by $\lambda$ and $X_{\alpha}$ acts as zero for $\alpha \in \tilde{\Pi} \Delta^{+} \backslash\{\delta, 2 \delta\}$. By the transitivity of induced modules, we have

$$
Z_{\chi}^{\tilde{\Pi}}(\lambda) \cong U_{\chi}(\mathfrak{g}) \otimes_{U_{\chi}(\mathfrak{p})} Z_{\chi}^{\mathfrak{p}}(\lambda) .
$$

It follows from [Wang and Zhao 2009a, Section 6.5] that if $\lambda$ satisfies $\left(\lambda+{ }^{\tilde{\Pi}} \rho \mid \delta\right)^{p}-$ $\left(\lambda+{ }^{\tilde{\Pi}} \rho \mid \delta\right)=0$, then $Z_{\chi}^{\mathfrak{o s p}(1 \mid 2)}(\lambda)$ is reducible; hence $Z_{\chi}^{\mathfrak{p}}(\lambda)$ and so $Z_{\chi}^{\tilde{\Pi}}(\lambda)$ will be 
reducible. By Proposition 4.3, $\left(\lambda+\tilde{\Pi}^{\tilde{\Pi}} \rho \mid \delta\right)^{p}-\left(\lambda+{ }^{\tilde{\Pi}} \rho \mid \delta\right)$ divides ${ }^{\tilde{\Pi}} \Phi(\lambda)$, that is, $\left((\lambda \mid \delta)^{p}-(\lambda \mid \delta)\right)$ divides $\Phi^{\prime}$. For two such roots $\delta$ and $\delta^{\prime}$, note that $\left((\lambda \mid \delta)^{p}-(\lambda \mid \delta)\right)$ and $\left(\left(\lambda \mid \delta^{\prime}\right)^{p}-\left(\lambda \mid \delta^{\prime}\right)\right)$ are coprime if $\delta \neq \pm \delta^{\prime}$. Since for any such root $\delta$, either $\delta$ or $-\delta$ is in $\Pi^{\Pi} \Delta_{\overline{1}}^{+}$, and since the choice of such $\delta$ is arbitrary, we conclude that

$$
\prod_{\delta \in \Pi^{-} \Delta_{\overline{1}}^{+} \backslash \Pi^{\Pi} \bar{\Delta}_{\overline{1}}^{+}}(\lambda \mid \delta) \cdot \prod_{2 \delta \in \Pi_{\Delta_{\overline{0}}^{+} \backslash \Pi^{-} \bar{\Delta}_{\overline{0}}^{+}}}\left((\lambda \mid 2 \delta)^{p-1}-1\right) \text { divides } \Phi^{\prime} .
$$

Any odd root $\beta \in \bar{\Delta}_{\overline{1}}$ (of type (ii)) appears in some set of simple roots. The root vector $X_{\beta}$ generates an embedded $\mathfrak{s l}(1 \mid 1)$. Using arguments similar to those for type (iii) simple roots above, we can show that

$$
\prod_{\beta \in \Pi^{\Pi} \bar{\Delta}_{\overline{1}}^{+}}(\lambda \mid \beta) \quad \text { divides } \Phi^{\prime} .
$$

In the proof, we need an irreducibility criterion for $\mathfrak{s l}(1 \mid 1)$-baby Verma modules, which can be easily deduced from that for $\mathfrak{g l}(1 \mid 1)$-baby Verma modules as in [Wang and Zhao 2009b, Proposition 7.7].

For roots in $\bar{\Delta}_{\overline{0}}$ (of type (i)), in a similar but classical way — see [Rudakov 1970, proof of Proposition 6] - we can show that

$$
\prod_{\alpha \in \Pi \bar{\Delta}_{\overline{0}}^{+}}\left((\lambda \mid \alpha)^{p-1}-1\right) \quad \text { divides } \Phi^{\prime} .
$$

Finally, the proposition follows from a degree consideration and the fact that the three factors above are mutually coprime.

Theorem 4.6. A baby Verma module $Z_{\chi}^{\Pi}(\lambda)$ for $\lambda \in \Lambda_{\chi}$ is irreducible if and only if

$$
\prod_{\alpha \in \Pi^{\Pi} \Delta_{\overline{0}}^{+}}\left(\left(\lambda+{ }^{\Pi} \rho \mid \alpha\right)^{p-1}-1\right) \cdot \prod_{\beta \in \Pi_{\Delta_{\overline{1}}^{+}}}\left(\lambda+{ }^{\Pi} \rho \mid \beta\right) \neq 0 .
$$

Proof. Follows readily from Propositions 4.3 and 4.5.

Theorem 4.7. The algebra $U_{\chi}(\mathfrak{g})$ is a semisimple algebra if and only if $\chi$ is regular semisimple.

Proof. The argument, which uses the irreducibility criterion in Theorem 4.6, is pretty standard. We include it here just for the sake of completeness.

Since $\chi$ satisfies $\chi\left(X_{\alpha}\right)=0$ for each $\alpha \in \Delta_{\overline{0}}$, for any set of simple roots $\Pi$, the baby Verma modules $Z_{\chi}^{\Pi}(\lambda)$ for $\lambda \in \Lambda_{\chi}$ have unique irreducible quotients, and they form a complete and irredundant set of irreducible $U_{\chi}(\mathfrak{g})$-modules. Now by the Wedderburn theorem and a dimension counting argument, $U_{\chi}(\mathfrak{g})$ is semisimple if and only if all the baby Verma modules $Z_{\chi}^{\Pi}(\lambda)$ for $\lambda \in \Lambda_{\chi}$ are simple. By Theorem 4.6, $Z_{\chi}^{\Pi}(\lambda)$ being simple for all $\lambda \in \Lambda_{\chi}$ is equivalent to ${ }^{\Pi} \Phi(\lambda) \neq 0$ for all 
$\lambda \in \Lambda_{\chi}$, which in turn is equivalent to (a) $\left(\lambda+{ }^{\Pi} \rho\right)\left(H_{\alpha}\right) \notin \mathbb{F}_{p} \backslash\{0\}$ for all $\alpha \in \Delta_{\overline{0}}$ and (b) $\left(\lambda+{ }^{\Pi} \rho\right)\left(H_{\beta}\right) \neq 0$ for all $\beta \in \Delta_{\overline{1}}$.

Under the current assumptions, $\chi$ is regular semisimple if and only if $\chi\left(H_{\alpha}\right) \neq 0$ for all $\alpha \in \Delta$. If $\chi$ is regular semisimple, then it follows that for any $\lambda \in \Lambda_{\chi}$, $\lambda\left(H_{\alpha}\right) \notin \mathbb{F}_{p}$ for all $\alpha \in \Delta$ since $\lambda\left(H_{\alpha}\right)^{p}-\lambda\left(H_{\alpha}\right)=\chi\left(H_{\alpha}\right)^{p}$. In this situation, both (a) and (b) are true since $\Pi_{\rho}\left(H_{\alpha}\right) \in \mathbb{F}_{p}$ for any $\alpha \in \Delta$. Hence all $Z_{\chi}^{\Pi}(\lambda)$ are simple and $U_{\chi}(\mathfrak{g})$ is semisimple.

Conversely, if $\chi$ is not regular semisimple, then $\chi\left(H_{\alpha}\right)=0$ for some $\alpha \in \Delta$. Let us assume $\alpha \in \Delta_{\overline{0}}$ since the other case can be argued similarly. Then $\lambda\left(H_{\alpha}\right) \in \mathbb{F}_{p}$ for $\lambda \in \Lambda_{\chi}$. Since shifting the value of $\lambda\left(H_{\alpha}\right)$ by a number in $\mathbb{F}_{p}$ will still result in an element in $\Lambda_{\chi}$ (noting that the values of $\lambda\left(H_{\beta}\right)$ for some $\beta \in \Delta$ will be changing correspondingly), we may thus assume $\left(\lambda+{ }^{\Pi} \rho\right)\left(H_{\alpha}\right)=1$. Then ${ }^{\Pi} \Phi(\lambda)=0$ and $Z_{\chi}^{\Pi}(\lambda)$ is reducible by Theorem 4.6. Hence $U_{\chi}(\mathfrak{g})$ is not semisimple.

Remark 4.8. The "if" part of the theorem is a consequence of the super KacWeisfeiler conjecture (Theorem 3.5); see [Wang and Zhao 2009a, Corollary 5.7].

Also, for type I basic classical Lie superalgebras, Theorems 4.6 and 4.7 are consequences of an equivalence of categories between typical $U_{\chi}(\mathfrak{g})$-modules and typical $U_{\chi}\left(\mathfrak{g}_{0}\right)$-modules; see [Zhao 2009, Theorems 4.1 and 4.3].

\section{Acknowledgments}

I am very grateful to my advisor, Weiqiang Wang, for valuable suggestions and advice. I am deeply indebted to A. Premet and S. Skryabin for their influential ideas. I thank I. Gordon for helpful discussions.

\section{References}

[Bell 1990] A. D. Bell, "A criterion for primeness of enveloping algebras of Lie superalgebras", $J$. Pure Appl. Algebra 69:2 (1990), 111-120. MR 92b:17014 Zbl 0723.17011

[Friedlander and Parshall 1988] E. M. Friedlander and B. J. Parshall, "Modular representation theory of Lie algebras”, Amer. J. Math. 110:6 (1988), 1055-1093. MR 89j:17015 Zbl 0673.17010

[Jacobson 1962] N. Jacobson, Lie algebras, Interscience Tracts in Pure and Applied Mathematics 10, Wiley, New York, 1962. MR 26 \#1345 Zbl 0121.27504

[Jantzen 1998] J. C. Jantzen, "Representations of Lie algebras in prime characteristic", pp. 185235 in Representation theories and algebraic geometry (Montreal, 1997), edited by A. Broer et al., NATO Adv. Sci. Inst. Ser. C Math. Phys. Sci. 514, Kluwer, Dordrecht, 1998. MR 99h:17026 Zbl 0974.17022

[Jantzen 2004] J. C. Jantzen, "Nilpotent orbits in representation theory", pp. 1-211 in Lie theory, edited by J.-P. Anker et al., Progr. Math. 228, Birkhäuser, Boston, MA, 2004. MR 2005c:14055 Zbl 1169.14319

[Kac 1977] V. G. Kac, "Lie superalgebras", Advances in Math. 26:1 (1977), 8-96. MR 58 \#5803 Zbl 0366.17012 
[Liu et al. 1991] S. X. Liu, M. Beattie, and H. J. Fang, "Graded division rings and the Jacobson density theorem”, Beijing Shifan Daxue Xuebao 27:2 (1991), 129-134. MR 93a:16032 Zbl 0746.16031

[Premet 1995] A. Premet, "Irreducible representations of Lie algebras of reductive groups and the Kac-Weisfeiler conjecture”, Invent. Math. 121:1 (1995), 79-117. MR 96g:17007 Zbl 0828.17008

[Premet 2002] A. Premet, "Special transverse slices and their enveloping algebras", Adv. Math. 170:1 (2002), 1-55. MR 2003k:17014 Zbl 1005.17007

[Premet and Skryabin 1999] A. Premet and S. Skryabin, "Representations of restricted Lie algebras and families of associative $\mathscr{L}$-algebras", J. Reine Angew. Math. 507 (1999), 189-218. MR 99m: 17026 Zbl 0978.17011

[Rudakov 1970] A. N. Rudakov, "The representations of classical semisimple Lie algebras in characteristic p", Izv. Akad. Nauk SSSR Ser. Mat. 34 (1970), 735-743. In Russian; translated in Math. USSR, Izv. 4 (1970), 741-749. MR 42 \#1872 Zbl 0242.17005

[Scheunert et al. 1976] M. Scheunert, W. Nahm, and V. Rittenberg, "Classification of all simple graded Lie algebras whose Lie algebra is reductive, I", J. Mathematical Phys. 17:9 (1976), 16261639. MR 54 \#2742a Zbl 0335.17014

[Serganova 2008] V. Serganova, "Kac-Moody superalgebras and integrability", preprint, 2008, available at http://math.berkeley.edu/ serganov/papers/superk.pdf.

[Skryabin 2003] S. Skryabin, "Representations of the Poisson algebra in prime characteristic", Math. Z. 243:3 (2003), 563-597. MR 2004d:17027 Zbl 1054.17015

[Veřsfeǔler and Kac 1971] B. J. Veřsfeǔler and V. G. Kac, "The irreducible representations of Lie p-algebras”, Funkcional. Anal. i Priložen. 5:2 (1971), 28-36. In Russian; translated in Funct. Anal. Appl. 5 (1971), 111-117. MR 44 \#2793 Zbl 0237.17003

[Wall 1964] C. T. C. Wall, "Graded Brauer groups", J. Reine Angew. Math. 213 (1964), 187-199. MR 29 \#4771 Zbl 0125.01904

[Wang and Zhao 2009a] W. Wang and L. Zhao, "Representations of Lie superalgebras in prime characteristic, I", Proc. Lond. Math. Soc. (3) 99:1 (2009), 145-167. MR 2520353 Zbl 1176.17013

[Wang and Zhao 2009b] W. Wang and L. Zhao, "Representations of Lie superalgebras in prime characteristic, II: The queer series”, preprint, 2009.

[Zhang 2009] C. Zhang, "On simple modules for the restricted Lie superalgebra $\mathfrak{g l}(m \mid n)$ ", preprint, 2009. arXiv 0905.1383

[Zhao 2009] L. Zhao, “Typical blocks of Lie superalgebras in prime characteristic”, preprint, 2009. arXiv 0905.1760

Received September 3, 2009.

LEI ZHAO

DEPARTMENT OF MATHEMATICS

UNIVERSITY OF OKLAHOMA

NORMAN, OK 73019-0315

UNITED STATES

prescheme@ou.edu 


\title{
PACIFIC JOURNAL OF MATHEMATICS
}

\author{
http://www.pjmath.org \\ Founded in 1951 by \\ E. F. Beckenbach (1906-1982) and F. Wolf (1904-1989)
}

\section{EDITORS}

V. S. Varadarajan (Managing Editor)

Department of Mathematics

University of California

Los Angeles, CA 90095-1555

pacific@math.ucla.edu

Vyjayanthi Chari

Department of Mathematics

University of California

Riverside, CA 92521-0135

chari@math.ucr.edu

Robert Finn

Department of Mathematics Stanford University

Stanford, CA 94305-2125

finn@math.stanford.edu

Kefeng Liu

Department of Mathematics

University of California

Los Angeles, CA 90095-1555

liu@math.ucla.edu
Darren Long

Department of Mathematics

University of California

Santa Barbara, CA 93106-3080

long@math.ucsb.edu

Jiang-Hua Lu

Department of Mathematics

The University of Hong Kong

Pokfulam Rd., Hong Kong jhlu@maths.hku.hk

Alexander Merkurjev

Department of Mathematics

University of California

Los Angeles, CA 90095-1555

merkurev@math.ucla.edu
Sorin Popa

Department of Mathematics University of California

Los Angeles, CA 90095-1555 popa@math.ucla.edu

Jie Qing

Department of Mathematics

University of California

Santa Cruz, CA 95064

qing@cats.ucsc.edu

Jonathan Rogawski

Department of Mathematics

University of California

Los Angeles, CA 90095-1555

jonr@math.ucla.edu

\section{PRODUCTION}

pacific@math.berkeley.edu

\begin{abstract}
Silvio Levy, Scientific Editor Matthew Cargo, Senior Production Editor
\end{abstract}
ACADEMIA SINICA, TAIPEI

CALIFORNIA INST. OF TECHNOLOGY

INST. DE MATEMÁTICA PURA E APLICADA

KEIO UNIVERSITY

MATH. SCIENCES RESEARCH INSTITUTE

NEW MEXICO STATE UNIV.

OREGON STATE UNIV.

\section{SUPPORTING INSTITUTIONS}

STANFORD UNIVERSITY
UNIV. OF BRITISH COLUMBIA
UNIV. OF CALIFORNIA, BERKELEY
UNIV. OF CALIFORNIA, DAVIS
UNIV. OF CALIFORNIA, LOS ANGELES
UNIV. OF CALIFORNIA, RIVERSIDE
UNIV. OF CALIFORNIA, SAN DIEGO
UNIV. OF CALIF., SANTA BARBARA

UNIV. OF CALIF., SANTA CRUZ

UNIV. OF MONTANA

UNIV. OF OREGON

UNIV. OF SOUTHERN CALIFORNIA

UNIV. OF UTAH

UNIV. OF WASHINGTON

WASHINGTON STATE UNIVERSITY

These supporting institutions contribute to the cost of publication of this Journal, but they are not owners or publishers and have no responsibility for its contents or policies.

See inside back cover or www.pjmath.org for submission instructions.

The subscription price for 2010 is US \$420/year for the electronic version, and \$485/year for print and electronic.

Subscriptions, requests for back issues from the last three years and changes of subscribers address should be sent to Pacific Journal of Mathematics, P.O. Box 4163, Berkeley, CA 94704-0163, U.S.A. Prior back issues are obtainable from Periodicals Service Company, 11 Main Street, Germantown, NY 12526-5635. The Pacific Journal of Mathematics is indexed by Mathematical Reviews, Zentralblatt MATH, PASCAL CNRS Index, Referativnyi Zhurnal, Current Mathematical Publications and the Science Citation Index.

The Pacific Journal of Mathematics (ISSN 0030-8730) at the University of California, c/o Department of Mathematics, 969 Evans Hall, Berkeley, CA 94720-3840, is published monthly except July and August. Periodical rate postage paid at Berkeley, CA 94704, and additional mailing offices. POSTMASTER: send address changes to Pacific Journal of Mathematics, P.O. Box 4163, Berkeley, CA 94704-0163.

PJM peer review and production are managed by EditFLOW ${ }^{\mathrm{TM}}$ from Mathematical Sciences Publishers.

PUBLISHED BY PACIFIC JOURNAL OF MATHEMATICS

at the University of California, Berkeley 94720-3840

A NON-PROFIT CORPORATION

Typeset in LATEX

Copyright $(\mathrm{C} 2010$ by Pacific Journal of Mathematics 


\section{PACIFIC JOURNAL OF MATHEMATICS}

Volume $248 \quad$ No. $2 \quad$ December 2010

Topological description of Riemannian foliations with dense leaves

Jesús A. ÁlVAREZ LóPEZ and Alberto CANDEL

The nonexistence of quasi-Einstein metrics

JEFFREY S. CASE

Twisted symmetric group actions

AKINARI HoSHI and MING-CHANG KANG

Optimal transportation and monotonic quantities on evolving manifolds

305

HONG HUANG

Hopf structures on the Hopf quiver $Q(\langle g\rangle, g)$

HuA-Lin HuANG, YU Ye and QING ZHaO

Minimal surfaces in $S^{3}$ foliated by circles

Nikolai KuteV and VelichKa Milousheva

Prealternative algebras and prealternative bialgebras

XIANG Ni and CHENGMING BAI

Some remarks about closed convex curves

Ke OU and Shengliang PAN

Orbit correspondences for real reductive dual pairs

SHU-YEN PAN

Graphs of bounded degree and the $p$-harmonic boundary

Michael J. Puls

Invariance of the BFV complex

FLORIAN SCHÄTZ

Some elliptic PDEs on Riemannian manifolds with boundary

YANNICK SIRE and ENRICO VALDINOCI

Representations of Lie superalgebras in prime characteristic, III 